\title{
TinkerCad - A Web Based Application for Virtual Labs to help Learners Think, Create and Make
}

\author{
Radha Abburi ${ }^{1}$, Manne Praveena ${ }^{2}$, R.Priyakanth ${ }^{3}$ \\ 1,2,3Associate Professor, Department of Electronics and Communication Engineering, BVRIT HYDERABAD College of \\ Engineering for Women, Hyderabad, Telangana, India. \\ ${ }^{1}$ radha.a@bvrithyderabad.edu.in \\ ${ }^{2}$ praveena.m@bvrithyderabad.edu.in \\ 3 priyakanth.r@bvrithyderabad.edu.in
}

\begin{abstract}
Amid COVID-19 shutdowns, in-person lectures and live lab classes have been suspended worldwide. In this scenario, digital instruction and sources can be a feasible alternate. In view of this, Virtual labs are a great approach to the web-based digital learning. These labs are available round the clock and provide for realistic abilities of science to the learners. Circuit simulators like LTSpice, PSpice, EasyEDA Simulator, Proteus by Lab center etc are advanced platforms for developers and planners hoping to become familiar with the nuts and bolts of circuit plan and schematics. However, these simulation tools do not offer a platform for all the students under one roof and do not allow the instructor to monitor their work as they progress with circuit building and simulation. This is made possible with a web-based software called Tinkercad which is free to use and can run directly in your web browser. Educators can monitor what students plan and reproduce in a virtual classroom in Tinkercad. It enables hands-on journey and sense of working inside the actual lab environment and allows the user to hook a wide array of electrical components together, program an Arduino and simulate analog and digital behaviour. This paper describes a case study of use of this software for lab simulation and describe some of its limitations. Details of survey responses from around 300 students demonstrate the efficacy of the Tinkercad platform compared to other simulation software available for engaging students in learning key hands-on lab skills.
\end{abstract}

Keywords: Web based Application, Virtual components, Simulation tools, e-learning

\section{Radha Abburi}

Department of ECE, BVRIT HYDERABAD College of Engineering for Women, Hyderabad, Telangana, India. radha.a@bvrithyderabad.edu.in

Introduction: A Virtual Laboratory is a computer-based activity in which college students interact via a computer interface with experimental setup or other activities. Typical examples include a simulation of an experiment in which a student interacts with a programmed action and a remote-managed experiment in which a student interacts through a computer connect with a real device, while She/he is remote from it. Digital laboratories allow college students to carry out a variety of experiments that are difficult to conduct due to hazards in real laboratories. [1] As a result of not having to stick to specific hours to enter the laboratory or move from one place to another, it helps educators and college students save effort and time and makes it easier for them to make use of the new technologies. They encourage college students to perform practical experiments linked to theoretical courses that enable them to absorb programmes and provide experimental behaviour with flexibility. For circuit design, various platforms similar to Easy EDA, PartSim, Every Circuit, Falstad, Do Ciruits, LT Spice, PSpice, Proteus, etc. can be found whereby circuits can be built, layouts developed, circuit evaluation and simulation.

On these platforms, the main issue we face is that there is no provision for tracking student progress. Taking this as the essential criteria that we began to explore, which led us to a web-based platform that was accessible for analogue and digital circuit design known as Tinkercad. This supports the group of teachers by offering a forum where the work of students can be witnessed. Tinkercad is a simple 3D design and modelling software for anyone based on a browser, suitable for students of all ages and abilities. It is a website that helps you, along with your own fingertips, to create problems at a convenient time. It is free and runs with an internet connection on each device. Tinkercad is a web-based software application that allows teachers to create an account and to use a code to invite college students to join. This allows college students to display their external designs and share their designs with the Tinkercad Community worldwide.

Tinkercad- A software based on a free browser at its easiest:

Tinkercad is a computer-aided design (CAD) software program that assists individuals and organisations in the manufacturing and promotion industry. It also caters to the healthcare and architecture sectors. Freeware makes editing files easy, allowing you to recreate existing designs. Designers can add and modify old 2D and 3D plans, too. With Tinkercad, you can create new fashions 
using the programme's modifiable shapes and objects.[2] Then, Tinkercad gives you the ability to combine shapes, add gestures, and stack blocks. With these acts, you can make a 3D design that is essential in prototypes. You can also create circuits using Tinkercad's traces and adjustable shapes. The code blocks then assist you in designing advertisement campaigns through GIFs. Lastly, Tinkercad includes interface presets to help you get started with the software.

\section{A First experience of the circuits of Tinkercad}

Although Tinkercad is mostly well-liked for its 3D modelling, one thing for circuit designers, i.e. electronics laboratories, can also be fantastic. By providing some personalised circuit elements, also called circuit assemblies, they have built-in electronics into the Tinkercad.[3] An editor is provided to college students to click on and drag recent components, all the stuff they would consider as a digital component, design their own circuit, and simulate to see how the circuit behaves. In each coding and block form, it additionally provides a programming atmosphere. Tinkercad Circuits opens up the potential for electrical versatility in your 3D printing designs. To that end, inside the individual dashboard, you'll find an entire part devoted to circuit tasks. cNow the CAD environment comes with component models. This means that you can create a circuit and hardware model, and the circuit can be simply placed within the template before $3 \mathrm{D}$ printing is completed.

\section{Arduino with Tinkercad}

Tinkercad Circuits combines Tinkercad with Arduino circuitry. Arduino is an open-source digital prototyping platform that also provides microcontrollers that offer Tinkercad, which revolves around the simulation of Arduino circuits, an entirely new feature. Tinkercad Circuits helps anyone to digitally build and program Arduino projects without the need for real hardware. As soon as you want to make a circuit, you can use the newest world of Tinkercad Circuits. You will see a bunch of digital elements drag-and - drop on the correct facet of the screen. At the top, you will be able to search and browse through a vast amount of accessible pieces. From LEDs to built-in circuits (ICs), and even a range of instrument tools, there's all small.[4] The open building space is the location where your design is intended. The toolbar on the top gives you the general operations for rotating, removing and even making notes in your different components beginning on the left. A cool characteristic is that, along with exporting and sharing your job, you will be able to get the component list. This makes it easier to put your creations into the real world.

Once you have a programmable item in your template, click on the button at the top of the toolbar to open the Code Viewer. The one-two gadgets available at present are the Arduino Uno R3 and the ATTiny. (ATTiny is an extra restricted and miniaturised Arduino). A simplified built-in integrated development environment ( IDE) is the programming space, which makes it very easy to programme the Arduino. The default approach is code blocks, and a twin view can be used to figure out how code blocks convert into single code. [5]

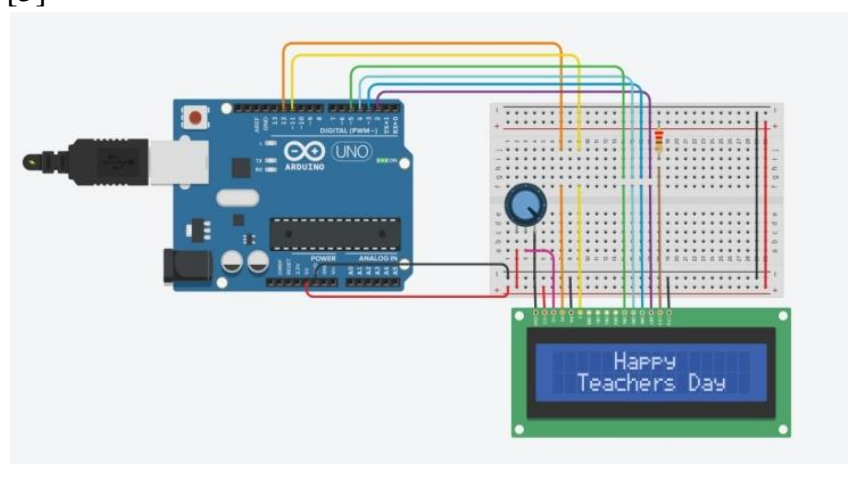

Fig 1: Sample Arduino Circuit created by students to wish all the teachers on Teacher's day Virtual celebrations

Therefore, they have the option of both designing 3D models, circuits, code blocks, and initially checking the lessons when learners $\log$ in to the Tinkercad website. With the 3D modelling tool, they create a 3D printable model, create circuits, simulate them, and even do the encoding with the circuit tool. The code-blocks allow students to create $3 \mathrm{D}$ designs using visible programming. They will explore ways to mount components, wire components, change components, and open classes to simulate them. This is merely self-learning. Students are in a position to follow and easily comprehend the instructions. Numerous components can be discovered and students can simply build their circuit. Apart from the supplies, there are some starters which are very helpful. In these starters, all students can do is simulate them, circuit design, and coding is already completed. Its programming environment is fantastic. Students have the option of either writing the code with block programming within the form of textual material, or each of them will be shown at a time. In addition, students can share their definition with various individuals and allow them to formulate the modifications.

\section{Tinkercad, why?}

The Tinkercad platform offers several advantages over various circuit simulation instruments. There's no need to instal anything on the computer, open source software, no licence needed, and an excellent circuit design and simulation interface and provision for student work monitoring. 
Involvement: For hours, Tinkercad will participate, whether students work on a specific design task or follow the built-in lessons; teachers should be prepared to step in and observe students' design and community interactions.

Pedagogy: Through a series of short, simple, and engaging lessons, Tinkercad immediately introduces students to its instruments and primes students to engage in its online community.

Help Offered: Jumping right in or taking someone else's project and tinkering with it is fast. It will take some reaching out to the community and support forum to get individual help.

All of these choices have led to the use of Tinkercad as a digital laboratory platform for pupils. All circuit laboratories, such as the Electronic Devices \& Circuits Lab, Digital System Design Lab, Electric Circuits Lab, Analog \& Digital Electronics Lab, etc., have been developed using the Tinkercad platform. A class has been generated for every lab and the class code has been shared with the students. Using this class code, students have to join the class and once they start designing circuits on the Tinkercad platform, it enables teachers to control their designs and recommend changes, check the validity of the design and assess accordingly.

Fig 2: Creating a class for the students

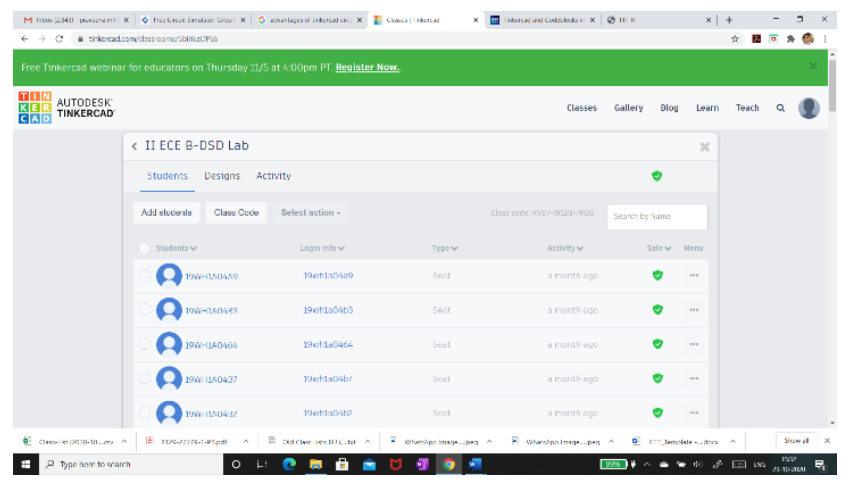

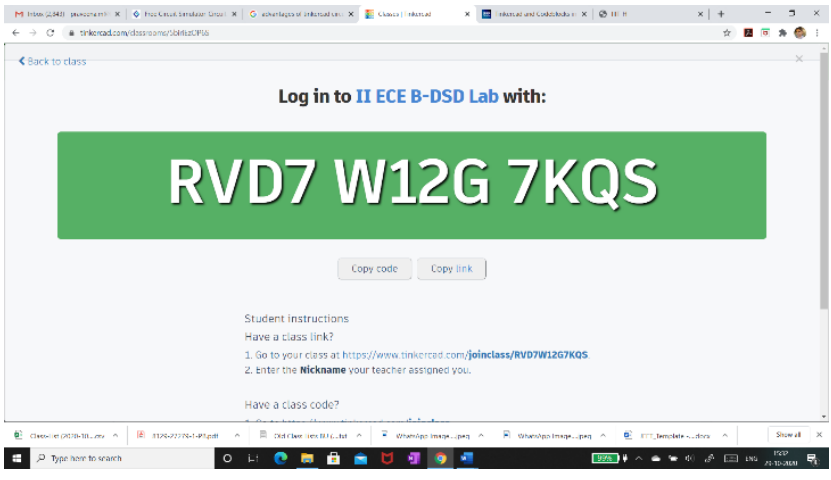

Fig 3: Class code to be shared with students

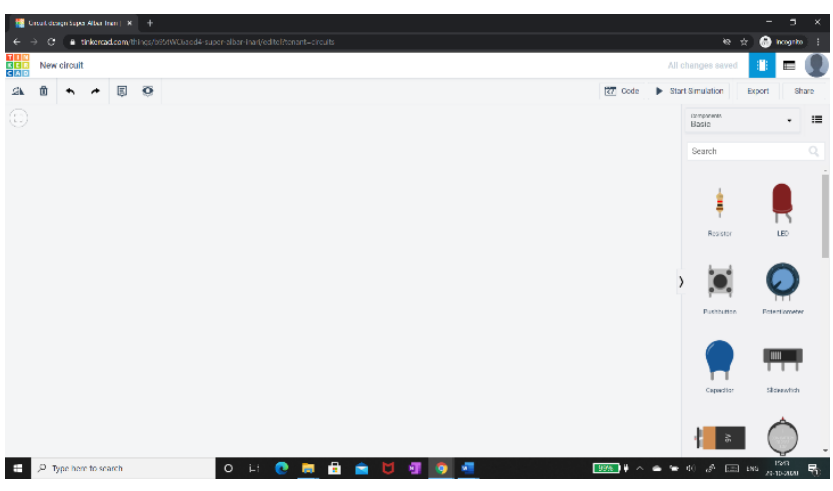

Fig 4: Open Building Area to design circuit

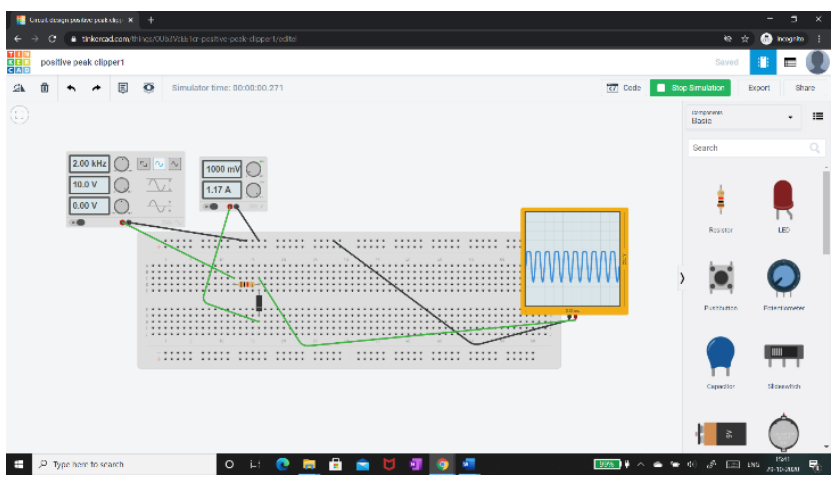

Fig 5: Circuit of positive peak clipper in Tinkercad

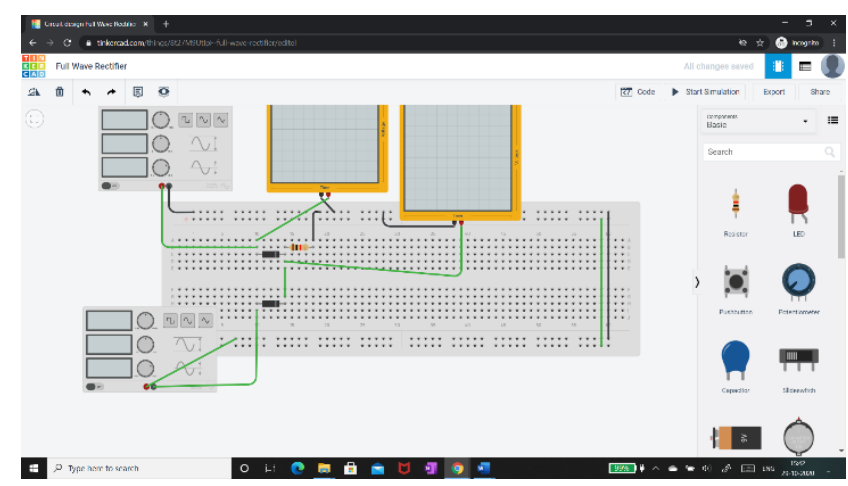

Fig 6: Circuit of Full Wave Rectifier in Tinkercad before simulation 


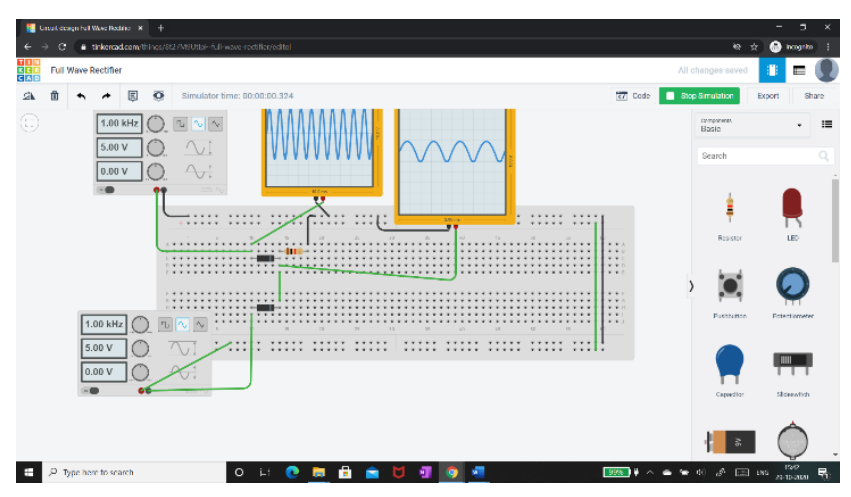

Fig 7: Circuit of Full Wave Rectifier in Tinkercad after simulation with output waveform

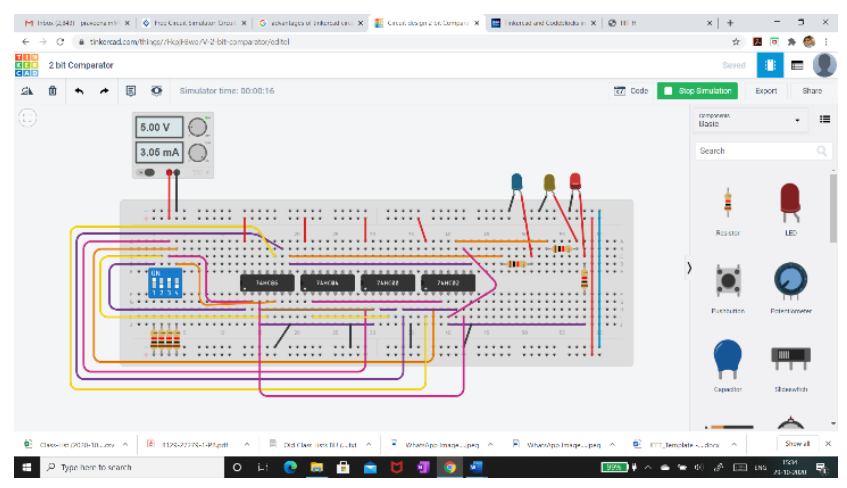

Fig 8: Circuit of a 2 bit comparator using logic gates in Tinkercad
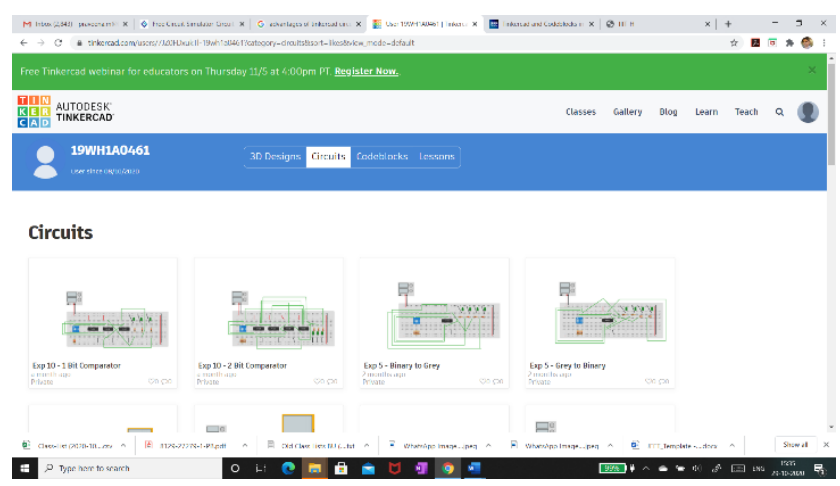

Fig 9: Student dashboard as viewed by Teacher

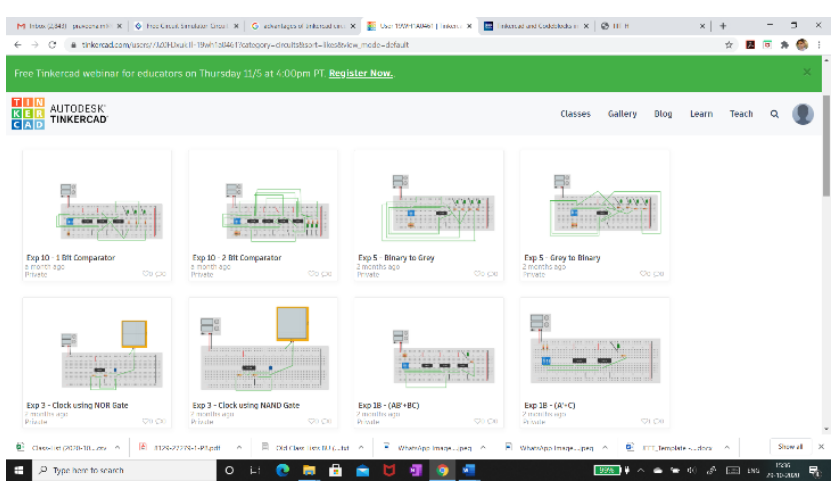

Fig 10: Various digital circuits designed by students in Tinkercad

\section{Transforming study halls into learning research centers:}

There may be every possibility that several questions come up within the minds of students through the lecture. For example, if the teacher while explaining the circuit shows the expected output for different values of the input, students may wonder if it actually happens the way it was explained. In this regard, if the instructor might allow students at the end of the session to confirm the output virtually on a digital platform like tinkercad, students can then and there open the browser, construct the circuit to simulate and examine whether or not the output is aligned with the idea discovered in theory. This course would but also assist students to know the idea by evaluating what they've learnt with what they've seen virtually without having to anticipate the following lab session to get their doubts clarified if any. This could additionally aid teachers to perceive the students' degree of understanding and provide speedy suggestions about their performance based on which students who excel are given a tougher set of circuits to work through, whereas those that are struggling are given extra primary circuits for practice. [6]

\section{Teachers' opinion:}

The "Fiddle with Virtual Components" Faculty Development Program has been conducted with 146 participants from 5 different states to explore different options available in TinkerCad to effectively foster undergraduate students to design analog \& digital circuits and projects.[7] 


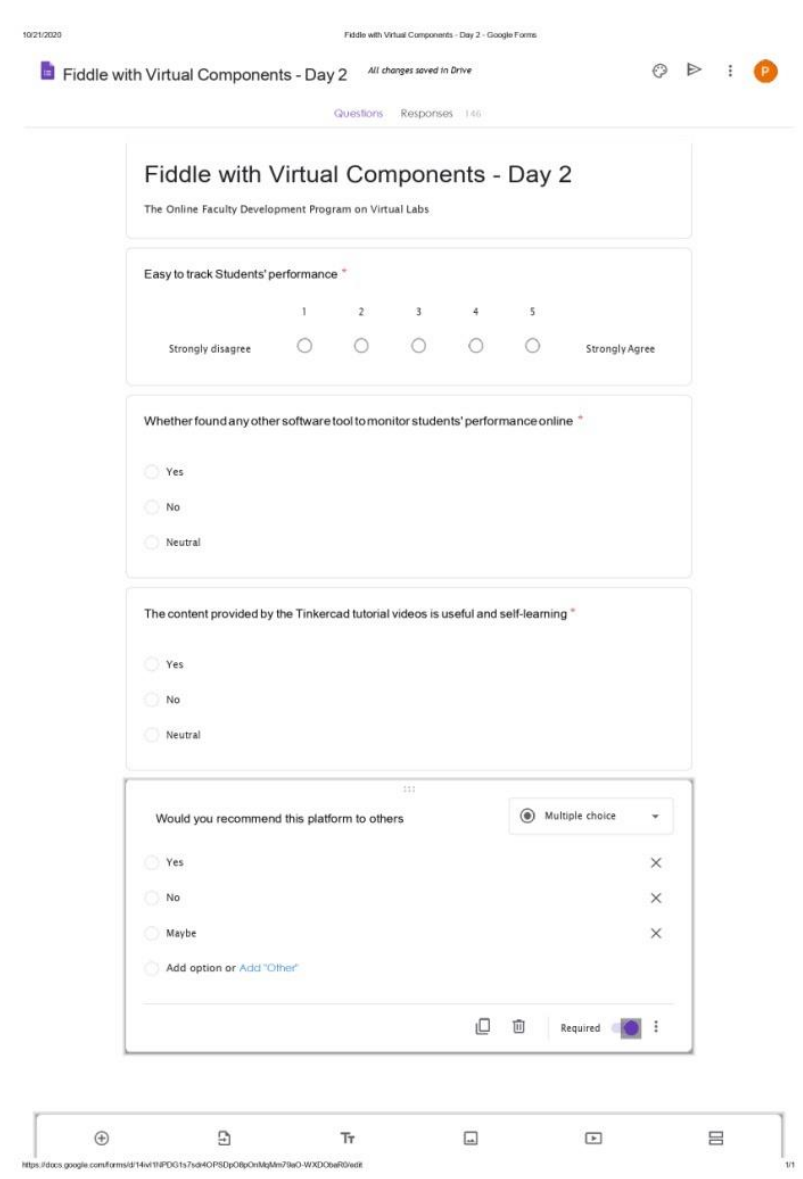

Fig 11: Sample Google Form shared with the participants

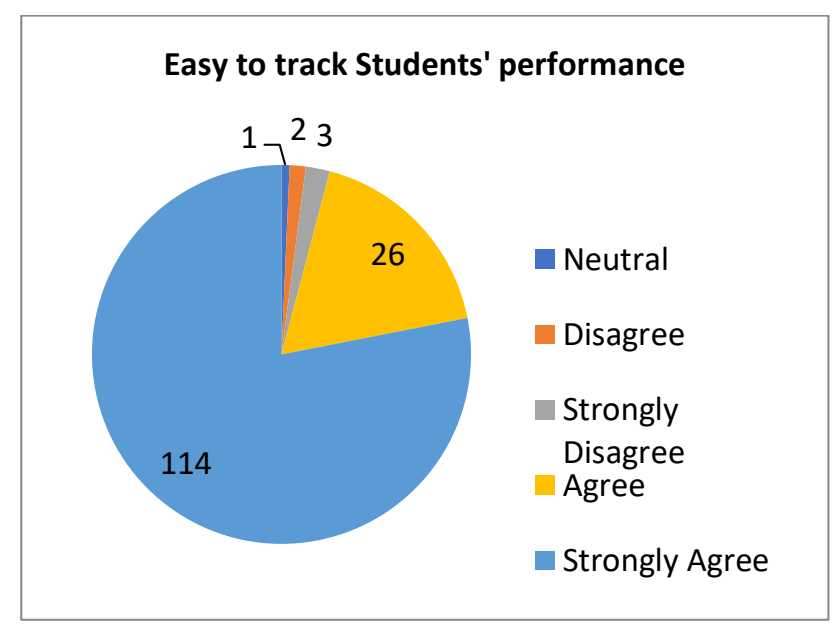

From the above figure, it is clear that 114 participants strongly agree that tracking the students ' progress and monitoring their work online is very easy.

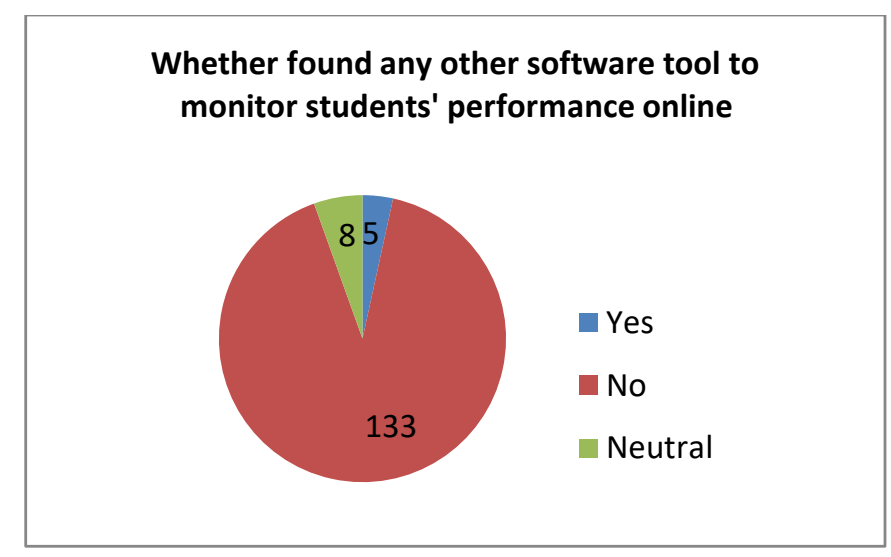

At the end of the Faculty Development Program, almost all participants came to the conclusion that there is rarely any forum for tracking the work of students online.

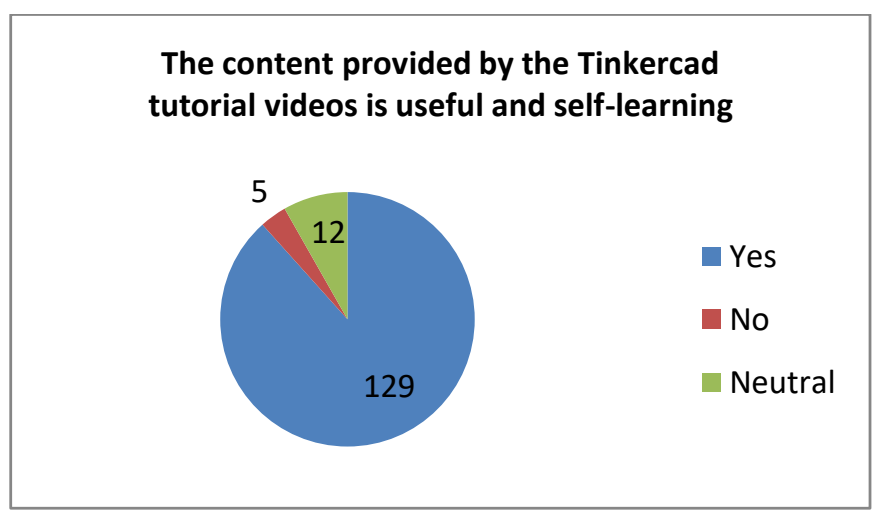

Teachers found that the tutorial videos on the tinkercad website were very beneficial to the students and helped with self-learning and also saved teachers a lot of time in preparing the learning material for the students and can thus focus more on the results of the students.

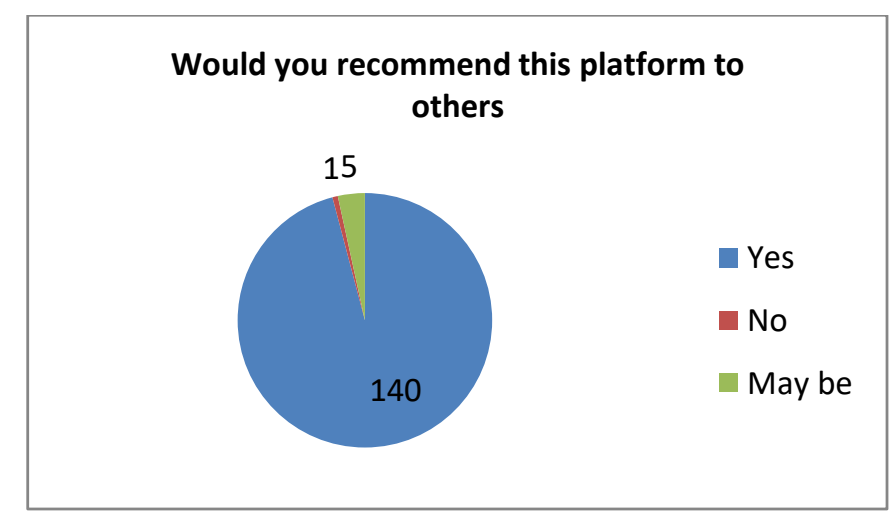

Analysis found that all teachers are excited about their laboratory sessions using the platform and suggest the platform to their peers as well.

\section{Students' Opinion:}

The survey responses from about 300 students are taken to demonstrate the usefulness of the Tinkercad platform in continuous monitoring of student work online compared to other simulation software available for engaging students 
in learning key hands-on laboratory skills. A google form is shared with the students with the following items.

1. Which among the softwares below does not need any assistance?

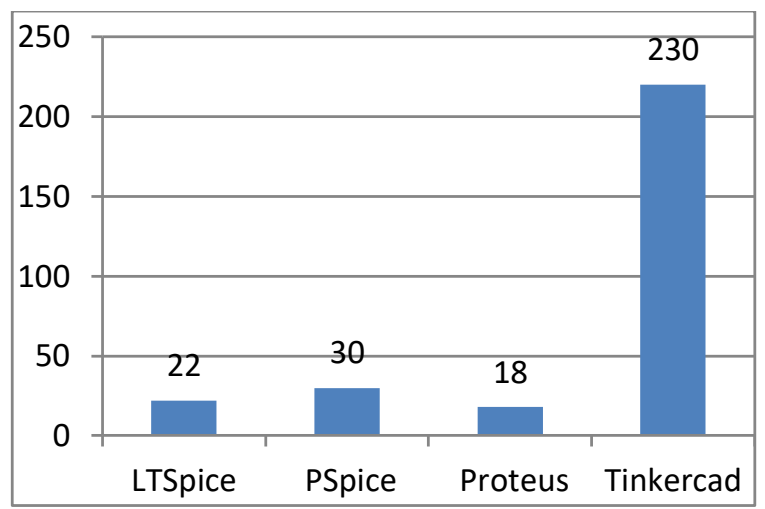

The above figure clearly shows that majority of the students have given their opinion that Tinkercad is self learning platform

2. Which facilitates individual and collaborative work amongst the following?

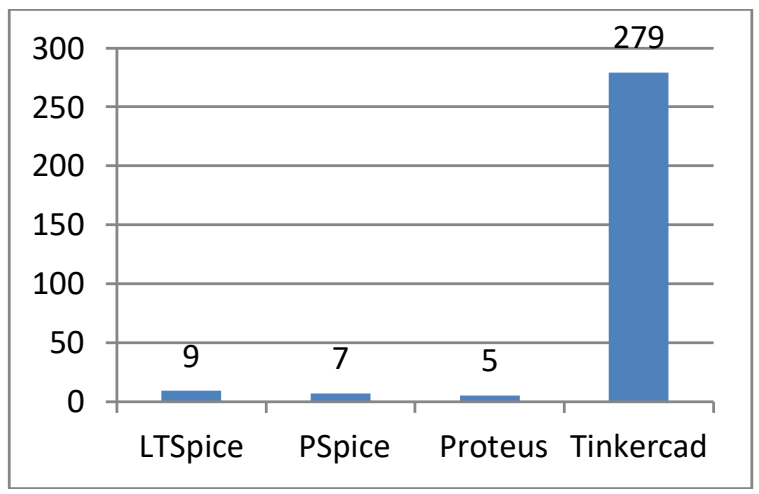

The above graph clearly states that according to majority of students Tinkercad facilitates individual and collaborative work

3. The platform which offers engaging lessons to learn how to create and simulate a circuit using the tools available.

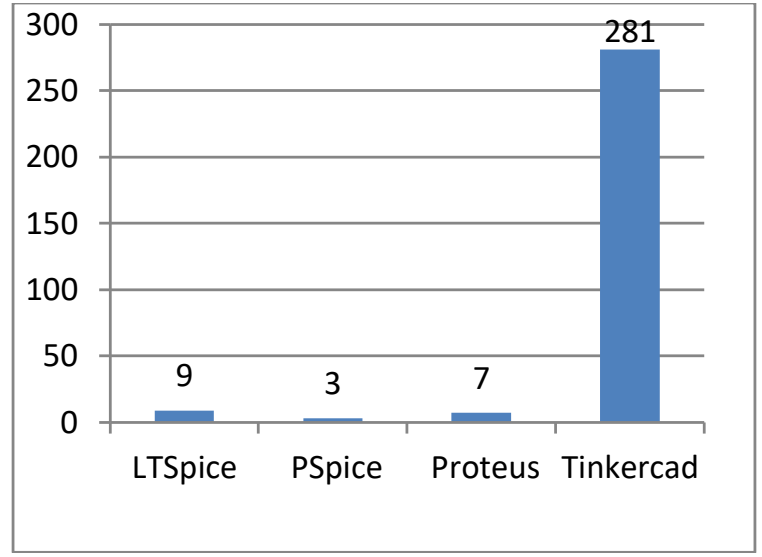

The above figure shows that Tinkercad offers many learning resources within the platform so that Students do not require separate demo sessions to be introduced by the teacher.

4. Suggest one among the following that permits to work on someone else's circuit

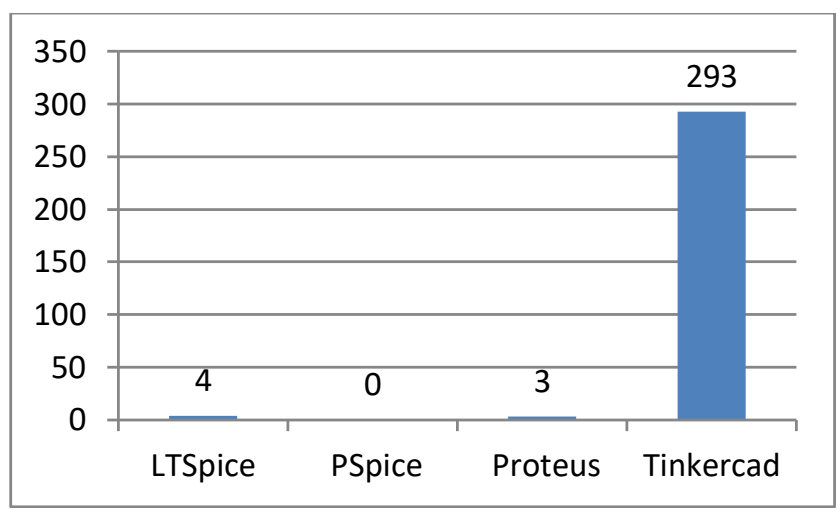

Majority of the students according to the above figure say that the oplatform also allows to work on someone else's circuit

5. Platform that allows students to participate in online community for any support

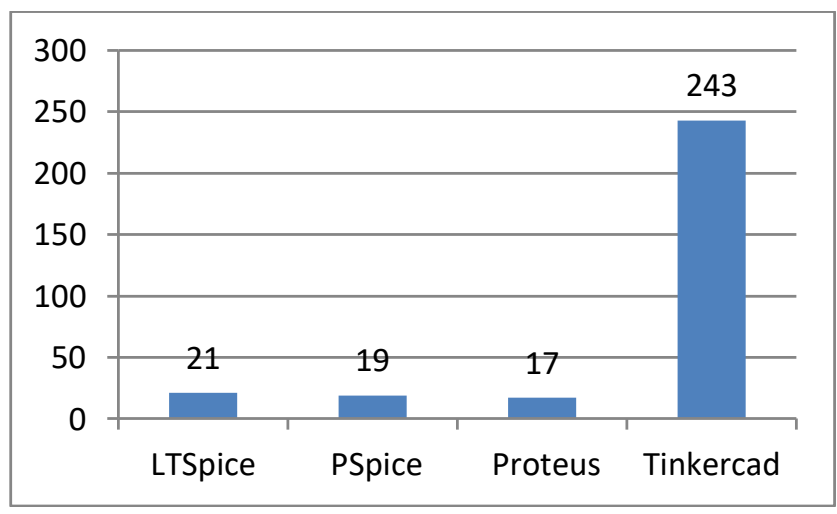

The above figure shows that the Tinkercad platform allows students to participate in online community for any support 
6. Platform which facilitates teachers to step in and observe the design and group experiences of students.

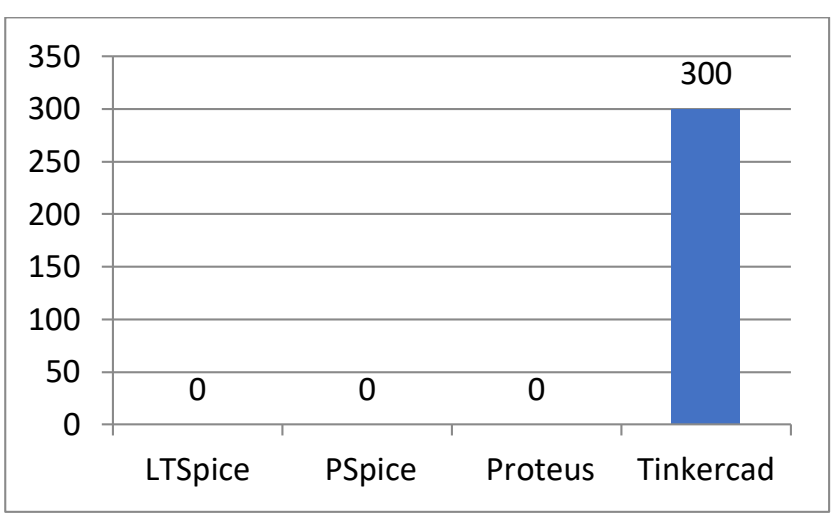

From the review of the study, it is obvious from the opinion of the students that almost all of the items in the list had a positive influence on the use of Tinkercad, a free web-based software, which is the best choice for both students and teachers. According to the opinion of students, it is also clear that Tinkercad is the only platform among the applications students are familiar with that enables teachers to track their work and success online continuously-

\section{Final Reflections:}

- There are numerous components to choose from, however it might all the time be more.

- Microcontroller simulation and easy circuitry work well, but generally, it can't deal with even comparatively easy analog circuits.

- Simple to memorize and use in general. good graphics and animations. Undo/redo capabilities for coding in addition to extra window resizing prospects can be nice.

\section{Restrictions:}

It needs an internet connection and a laptop or desktop, which for tablet and BYOD (Bring Your Own Device) environments could be a problem.

\section{Future Scope}

Tinkercad Circuits opens up the opportunity of electrical performance in the $3 \mathrm{D}$ printing projects. Its $\mathrm{CAD}$ setting now comes with circuit part models. This implies you'll be able to design a model with circuits and hardware, and as soon as $3 \mathrm{D}$ printed, the circuit may be simply positioned within the creation.

\section{Acknowledgement}

We appreciate the support from the Head of the Department, ECE, Principal and Management of Sri Vishnu Educational Society for providing the required facilities for conducting various activities for both teachers and students to explore various options available in TinkerCad to help students effectively towards designing the analog \& digital circuits and projects.

\section{References}

1. The virtual laboratory and interactive screen experiments Paul A Hatherly The Open University.

2."TINKERING WITH TINKERCAD A Beginner's Guide to Creating 3D Printer Designs" Michael Hibben \& Sarah Holmes

3. Electronic Circuit Basics With Tinkercad By Alex Reyes

4."EFFECTIVENESS OF E-LAB USE IN SCIENCE TEACHING AT THE OMANI SCHOOLS" TOJET: The Turkish Online Journal of Educational Technology January 2015, volume 14 issue 1

5.TMP36 Temperature Sensor With Arduino in Tinkercad. https://www.instructables.com/TMP36Temperature-Sensor-Arduino-Tinkercad/

6. Getting Started in Tinkercad By Bonnie Roskes, 3DVinci

7. Case Study Tinkering with Teachers: The Case for 3D Printing in the Education Library RACHAEL ELROD1 University of Florida, Gainesville, FL

8. "eLab - Remote electronics lab in real time" November 2007 Proceedings - Frontiers in Education Conference DOI: $10.1109 /$ FIE.2007.4418154

SourceIEEE Xplore

9. Tutorial TinkerCAD Electrical Series Circuit By: Matthew Jourden Brighton High School,Brighton, MI

10. Achuthan, K., Kolil, V. K., \& Diwakar, S. (2018). Using virtual laboratories in chemistry classrooms as interactive tools towards modifying alternate conceptions in molecular symmetry. Education and Information Technologies, 23,2499-2515.

https://doi.org/10.1007/s10639-018-9727-1.

11. Campbell, B., Collins, P., Hadaway, H., Hedley, N. and Stoermer, M. (2002). Web3D in Ocean Science Learning Environments: Virtual Big Beef Creek. In Proceedings of the 2002 Web3D Symposium HTML Abstract/ PDF Full paper

12. Jensen, N., Seipel, S., Nejdl, W. \& Olbrich, S. (2003) CoVASE --Collaborative Visualization for Constructivist Learning. CSCL Conference 2003, (pp. 249-253).

13. Jensen, N., Seipel, S., von Voigt, G., Raasch, S., Olbrich, S. \& Nejdl, W. (2004). Development of a Virtual Laboratory System for Science Education and the Study of Collaborative Action. In P. Kommers \& G. Richards (Eds.), Proceedings of World Conference on Educational Multimedia, Hypermedia and Telecommunications 2004 (pp.2148-2153).Chesapeake,VA:AACE.

Abstract/HTML/PDF

14.http://www.olimpicolearning.org/uploads/4/2/8/8/4288 4059/3d_printing_and_design_gr_6-12.pdf

15. https://promoambitions.com/tinkercad/

16. Website review by Marianne Rogowski, Common Sense Education | Updated September 2018 https://www.commonsense.org/education/website/tinkerc $\mathrm{ad}$ 